\title{
Gestion des travailleurs seniors : le rôle de la communication organisationnelle âgiste
}

\section{Martine Lagacé}

\section{(2) OpenEdition}

12 Journals

Édition électronique

URL : http://journals.openedition.org/communicationorganisation/659

DOI : 10.4000/communicationorganisation.659

ISSN : $1775-3546$

Éditeur

Presses universitaires de Bordeaux

\section{Édition imprimée}

Date de publication : 1 décembre 2008

Pagination : 202-220

ISBN : 978-2-86781-582-9

ISSN : $1168-5549$

\section{Référence électronique}

Martine Lagacé, « Gestion des travailleurs seniors : le rôle de la communication organisationnelle âgiste », Communication et organisation [En ligne], 34 | 2008, mis en ligne le 01 décembre 2011, consulté le 19 avril 2019. URL : http://journals.openedition.org/communicationorganisation/659 DOI : 10.4000/communicationorganisation.659 
Analyses

\section{Résumé}

Les travailleurs de 45 ans et plus représentent une part substantielle de la population active, lesquels pourraient pallier au défi des pénuries de maind'œuvre. Cependant, pour générer une extension de la vie professionnelle, le discours négatif sur l'âge doit être remplacé par celui d'une revalorisation de l'expérience. Dans cet article, nous montrons comment les croyances âgistes sont coûteuses pour le travailleur senior et pour l'organisation. Ce faisant, nous abordons le rôle de la communication organisationnelle en tant que véhicule de l'âgisme, tout autant que celui du gestionnaire, comme élément clé dans la modification du discours sur le vieillissement au travail.

\section{Mots-clefs}

Âgisme, communication organisationnelle, travailleurs seniors, désengagement, gestion

\section{Abstract}

Workers aged 45 years old and over represent a substantial part of the population, which could overcome the challenge of shortages of labor. However, in order to generate an extension of professional life, the current negative discourse on age must be replaced by one valuing experience. In this article, we show how ageist beliefs are costly for the senior worker as well as for the organization. In doing so, we explore the role of organizational communication as a vehicle of ageism, as well as the manager, as a key element in changing the discourse on aging at work.

\section{Key-Words}

Ageism, organizational communication, ageing workers, disengagement, management

Martine Lagacé est Professeure adjointe au Département de communication de l'Université d'Ottawa. 
Gestion des travailleurs seniors...

\section{Gestion des travailleurs seniors : le rôle de la communication organisationnelle âgiste \\ Martine Lagacé}

mmlagace@uottawa.ca

\section{Le contexte social et organisationnel du vieillissement}

L'augmentation de la longévité change profondément les traits d'une société. En Amérique, comme dans l'ensemble des pays de l'OCDE où la révolution démographique de l'avancement en âge se fait sentir (OCDE, 2006), la famille, par exemple, porte les traces indélébiles de ce changement. Celle-ci est en effet recomposée non plus seulement par des liens de diverses fratries mais aussi par des liens de multiples cohortes générationnelles: arrière petit-fils/petites-filles et arrières grands-parents ne sont plus des phénomènes hors du commun (ce qui n'implique pas nécessairement que ceux-ci se côtoient davantage mais, à tout le moins, ils en ont désormais l'opportunité). En outre, les organisations de travail sont aussi redessinées, redéfinies par les bouleversements démographiques : la rentrée tardive sur le marché du travail des jeunes, conjuguée au vieillissement global de la maind'œuvre active (OCDE, 2006; RHDSC, 2007) imposent à ceux et celles qui gèrent cette main-d'œuvre, le défi de la diversité des âges. Ce défi peut être perçu soit comme une «occasion» de réinventer le travail, soit comme un «problème» à résoudre. Plus souvent qu'autrement, c'est la seconde interprétation, négative, qui a prépondérance et ce particulièrement en ce qui a trait à la gestion des travailleurs âgés. Plusieurs études ont en effet montré que nombre d'entreprises ont souvent tendance à avoir recours à des politiques d'éviction de leur main-d'œuvre vieillissante plutôt que de maintien en emploi de celle-ci (Côté, 1991; McDonald \& Wanner, 1987). Guérin et Saba (2003) soulignent à cet effet certaines pratiques mises de l'avant dans les années 80 et 90 telles que les primes de départ, les programmes de préparation à la retraite, la diminution des responsabilités, lesquelles visaient à accélérer le départ à la retraite des seniors.

Les organisations de travail, comme éléments microcosmes d'une société, sont le reflet des croyances et des perceptions véhiculées sur l'avancement en âge, à plus large échelle. Le vieillissement, la vieillesse, la personne âgée, son rôle, sa place dans une société demeurent des sujets tabous dans les sociétés industrialisées, 
Analyses

lesquelles nourrissent plutôt un culte à la jeunesse (Glover \& Branine, 2001). Par exemple, les sphères médiatiques et politiques passent outre les sujets liés de près ou de loin au vieillissement et quand ceuxci sont abordés, ils le sont plus souvent qu'autrement dans l'écho d'un discours foncièrement négatif (AIFA, 2002). Les représentations sociales de la personne âgée sont en effet connotées de stéréotypes négatifs tels que la maladie, le déclin, la laideur, la décrépitude, pour ne nommer que ceux-là (Palmore, 2001 ; 2005a; 2005b) ; et quoique des stéréotypes positifs soient aussi utilisés pour dépeindre les aînés, plus souvent qu'autrement, ceux-ci réfèrent à leur "gentillesse », leur «personnalité chaleureuse » alors que leur «compétence » (dans le sens d'autonomie, de capacité, de confiance, etc.) est sérieusement remise en question (Cuddy \& Fiske, 2002).

En somme, la question du vieillissement et par extension celle de l'aîné sont des sujets peu prisés au plan social et quand ils le sont, c'est bien souvent avec une connotation de pessimisme, voire de pitié. Comment s'étonner alors que le travailleur senior, évoluant dans une organisation microcosme de la société, soit aussi dépeint sur la base de préjugés et de stéréotypes négatifs ? Productivité en déclin, résistance au changement, capacité réduite quant à l'apprentissage et à l'adaptation - particulièrement face aux nouvelles technologie -, incompétence, taux d'absentéisme plus élevé par rapport aux plus jeunes travailleurs (Arrowsmith \& McGoldrick, 1996; Avolio \& Barrett, 1987 ; Taylor \& Walker, 1993, 1994, 1996), n'en sont que quelques exemples. En outre, entre ces représentations négatives et la concrétisation de ces dernières, le pas est vite franchi : le travailleur vieillissant a moins accès à la formation continue, moins de chances de promotion que son plus jeune collègue et une fois au chômage, ses chances d'embauche sont considérablement réduites (Congrès du travail du Canada, 2004 ; RHDSC, 2003).

\section{Le paradoxe de la réalité démographique et des pratiques de gestion des travailleurs seniors}

La vision et les attitudes négatives à l'égard du vieillissement au travail sont d'autant plus paradoxales en regard des projections démographiques. Les statistiques révèlent en effet que des pénuries de main-d'œuvre et de compétences sont anticipées (OCDE, 2006 ; RHDSC, 2007), lesquelles se sont déjà actualisées dans plusieurs secteurs de travail (la santé, l'éducation pour ne nommer que ceux-ci), particulièrement au niveau des postes de gestion (Cowan, Farquhar, MacBride-King, 2002). Or, du même coup, les travailleurs seniors 
(âgés de 45 ans et plus ${ }^{1}$ ) représentent, à l'heure actuelle, un bassin de main-d'œuvre important, en termes de nombre; et cette force de main d'France est sans aucun doute plus scolarisée et plus en santé que les précédentes cohortes de travailleurs seniors. En outre, nombre de ces travailleurs vieillissants s'inscrivent en porte à faux avec la logique (encore trop prisée) d'une sortie précoce, d'une retraite anticipée. Le travail, sphère centrale d'intégration dans les sociétés industrialisées (Gaullier, 1982, 2003), procure en effet à ces travailleurs, un sentiment d'accomplissement, d'utilité, de contribution ; c'est une voie par laquelle se concrétise leur expérience et leur expertise acquise et ce faisant, le retrait précipité de cette même sphère n'est pas, pour plusieurs d'entre eux, une option privilégiée (DRHC, 2007). D'autant que plusieurs chercheurs soutiennent que la génération actuelle de travailleurs seniors, largement issue du baby boom, en est une pour laquelle le travail, voire la réalisation de soi par le travail, est une valeur de vie fondamentale (Lancaster \& Stillman, 2002).

Les pratiques de gestion de la main-d'œuvre âgée des décennies 80 et 90 que nous avons évoquées plus haut, ont aussi contribué à nourrir le paradoxe à l'égard du vieillissement au travail. Ces pratiques traduisent en effet un double discours (Guérin \& Saba, 2003) : en même temps qu'était évoquée l'importance de mettre en place des mesures de rétention des travailleurs vieillissants pour prolonger leur vie professionnelle, la réalité consistait plutôt à encourager leur sortie anticipée, voire précipitée. Désormais, en raison de l'intensification des pénuries de main-d'œuvre et de compétence, l'intérêt pour la mise en place de pratiques de rétention des travailleurs âgés est plus marqué (Guérin \& Saba, 2003). Ce qui ne signifie pas pour autant que les croyances, les perceptions véhiculées sur le vieillissement dans les organisations aient changé ; et pourtant, ce n'est qu'au prix d'un tel changement de mentalité que l'on pourra véritablement parler de succès, à moyen comme à long terme, des pratiques de maintien en emploi des seniors.

1 «Dans tous les documents examinés (que ce soit ceux du Canada ou de certains pays de l'OCDE), on entend par "adultes âgés" ou "travailleurs âgés" les personnes de 45 ans et plus, 55 ans et plus ou 65 ans et plus. Aux fins du présent projet, et afin de simplifier les choses, par "travailleur âgé" on fera référence aux travailleurs âgés de 45 ans et plus, sauf indications contraires. » In Malatest \& Associates, 2003. 
Analyses

Relativement peu d'études empiriques se sont penchées sur les préjugés et stéréotypes négatifs à l'égard du travailleur senior et surtout sur leurs répercussions pour ce même travailleur, comme pour l'organisation et pour la société de manière générale. Le constat est encore plus vrai lorsqu'il s'agit d'études ciblant les mécanismes par lesquels sont véhiculés, voire maintenus, ces stéréotypes. Des chercheurs ont amorcé une réflexion en ce sens, en circonscrivant la communication organisationnelle comme l'une des courroies de transmission centrales au maintien de l'âgisme au travail (Giles, Coupland, \& Coupland, 1991 ; Giles, Fox, Harwood \& Williams, 1994 ; Giles \& Reid, 2005 ; McCann \& Giles, 2002). Cependant que les répercussions de la communication âgiste au travail, en termes de désengagement psychologique et effectif du travailleur senior, restaient à être démontrées, tout autant que l'identification de pistes de solution visant à contrer cette forme de communication. C'est dans cette optique que s'inscrivent les objectifs du présent article. Ainsi, à la lumière d'études antérieures, il s'agit d'abord $a$ ) de faire l'état des lieux quant aux préjugés et stéréotypes à l'égard du travailleur senior et de circonscrire ensuite le rôle de la communication organisationnelle à cet effet ; $b$ ) en outre, toujours dans la continuité d'études antérieures, nous démontrerons que la communication âgiste s'avère être le précurseur d'un processus long et complexe de désengagement du travailleur senior. Ce faisant, en ayant identifié les sources par lesquelles sont maintenus, voire renforcés, les préjugés sur la base de l'âge au travail, la dernière composante du présent article vise à c) identifier certains éléments d'une organisation qui permettraient de contrer de tels préjugés. En ce sens, les gestionnaires, les dirigeants, parce qu'ils donnent le ton dans l'organisation (par le biais de leurs messages verbaux comme non verbaux), représentent un des éléments centraux quant à la transformation de la représentation $\mathrm{du}$ vieillissement au travail.

\section{Les stéréotypes et préjugés à l'égard du travailleur senior et leurs répercussions}

À quel âge est-on vieux ou plutôt, est-on perçu comme vieux au sein d'une organisation de travail? Dans plusieurs secteurs de travail, notamment celui des nouvelles technologies, dès l'âge de 45 ans (ou aux environs de), un travailleur est considéré vieux (Finkelstein, Burke \& Raju, 1995 ; Taylor \& Walker, 1993, 1994, 1996). Un phénomène paradoxal d'ailleurs en ce qu'à l'heure actuelle, on devient physiquement vieux beaucoup plus tard mais socialement, beaucoup plus tôt. En soi, cela ne serait pas un problème si ce n'était de la représentation sociale accolée au vieillissement. Nous l'avons évoqué 
plus haut, vieillir dans les sociétés industrialisées n'est pas de tout repos. L'aîné est coincé entre ses aspirations individuelles, lesquelles s'inscrivent de plus en plus dans une logique d'avancement, d'engagement, de continuité, de développement (allongement de l'espérance de vie en bonne santé oblige) et le regard social posé sur le vieillissement duquel se dégage des attentes de désengagement, d'arrêt, de rupture. Tant et aussi longtemps que persisteront les préjugés et stéréotypes négatifs sur la base de l'âge, ce décalage entre le vieillir personnel et le vieillir social ne se creusera que davantage. En outre, ce décalage s'exprime tout autant dans une logique macro que micro: ainsi, dans une organisation, les travailleurs seniors doivent aussi faire face à des croyances âgistes quant à leurs habiletés, leur productivité, leur capacité de changement, d'adaptation; croyances qui, pour bon nombre de ces travailleurs ne correspondent aucunement à leur performance réelle (Salthouse \& Maurer, 1996). Du coup, l'une des façons de contrer ce décalage entre perception et réalité risque de coûter cher tout autant à l'organisation qu'au travailleur senior, en ce qu'elle sous-tend le désengagement de ce dernier et conséquemment, la perte d'un savoir pour cette même organisation. C'est ce suggèrent les résultats d'une série d'études menées auprès de travailleurs fonctionnaires $(\mathrm{N}=117)$ et de travailleurs du domaine de la santé $(\mathrm{N}=976)$, tous âgés de 45 ans et plus (Lagacé, Tougas, 2006 ; Lagacé, Tougas, Laplante, Neveu, 2008a ; Lagacé, Tougas, Laplante, Neveu, 2008b ; Tougas, Lagacé, Laplante, Bellehumeur, 2008). Ainsi, lorsque ces travailleurs seniors perçoivent être la cible de préjugés et de stéréotypes négatifs sur la base de l'âge, ils amorcent une forme de rupture psychologique, de retrait mental du travail et ce, à deux niveaux: a) quant à la pertinence, à la validité de la rétroaction sur leur performance au travail et b) quant à la valeur, à l'importance accordée au travail. Le premier mécanisme de désengagement psychologique réfère au discrédit, le second à la dévaluation (Crocker \& Major, 1989; Crocker, Major, \& Steele, 1998 ; Major \& Schmader, 1998, 2001). En outre, ces mêmes études révèlent que cette stratégie de désengagement est dommageable pour le bien être psychologique du travailleur : son estime de lui-même s'en trouve en effet réduite. Cependant, les effets $\mathrm{du}$ désengagement psychologique du travailleur vieillissant sont d'autant plus perturbants qu'ils se ramifient dans des comportements de repli professionnel, voire de retrait effectif : l'équipe de Lagacé et $a l$., (2008b) suggère en effet que ce retrait mental, cette coupure virtuelle du travail qu'est le désengagement psychologique assombrit non seulement l'estime de soi des travailleurs vieillissants mais les conduit à des intentions effectives, concrètes de départ. C'est ici que 
les préjugés trouvent écho dans des comportements bien tangibles et c'est aussi à ce moment que l'organisation paie le prix du désengagement des seniors en ce que se volatilisent l'expertise, l'expérience, le savoir et la mémoire.

Dans le contexte actuel de pénuries de main-d'œuvre et de compétences, la mémoire du savoir faire et du savoir être est probablement ce qu'il y a de plus précieux pour l'organisation. Faire en sorte que cette mémoire de travail ne se désagrège pas devient une question de survie organisationnelle. En outre, cela sous-tend un travail colossal en ce qu'il s'agit de contrer les préjugés et stéréotypes âgistes, tout autant que le traitement différentiel sur la base de l'âge qui, bien souvent, en découle. Ce dont il est question en fait c'est d'un changement de mentalité qui doit s'opérer et qui permettra la mise en place d'une culture de travail inclusive et non plus exclusive. Le premier pas vers la création de cette culture inclusive pour tous les âges consiste à saisir les sources, les voies par lesquelles s'expriment, se canalisent les stéréotypes et préjugés âgistes au travail. Le présent article ne permet certainement pas de circonscrire la totalité de ces voies mais à tout le moins d'en cibler une qui semble jouer un rôle central et surtout sur laquelle il est possible d'agir : la communication organisationnelle.

\section{La communication organisationnelle comme véhicule des préjugés} et des stéréotypes sur la base de l'âge

Le rôle de la communication dans son lien avec les attitudes âgistes au travail est encore trop peu exploré. Pourtant, le discours, verbal tout autant que non verbal, constituent de solides assises sur lesquels sont véhiculés les préjugés face au vieillissement. Cela n'a rien d'étonnant en ce que la communication guide nos perceptions en même temps qu'elle est modelée, qu'elle traduit ces mêmes perceptions (Morreale, Spitzberg, \& Barge, 2001). Lorsqu'un individu communique, il le fait à partir d'un système de perceptions, lequel est sélectif et se base sur des éléments d'information les plus saillants quant à l'identité de son interlocuteur. Lorsque la valence associée à des éléments d'information saillants est plutôt négative, le discours utilisé (verbal comme non verbal), n'est que le reflet de cette négativité. Ce processus, que l'on pourrait en quelque sorte qualifier d'ajustant (cela non pas nécessairement au bénéfice de l'interlocuteur) est central dans la Théorie de la communication accomodante (Giles \& Reid, 2005 ; Giles, Coupland \& Coupland, 1991).

Cette théorie s'inscrit dans une perspective de relations intergroupes selon laquelle l'appartenance à un groupe social (en l'occurrence celui 
des travailleurs seniors), serait l'élément déterminant en fonction duquel toute la dynamique de la communication se construirait. Précisément, l'échange communicationnel entre deux individus serait dérivé de la reconnaissance de l'identité respective de ceux-ci, l'un percevant l'autre d'abord et avant tout en fonction des caractéristiques relatives à son groupe d'appartenance, les plus saillantes étant liées à la race, au sexe, à la religion et à l'âge (Cuddy \& Fiske, 2002). Par exemple, la reconnaissance, par l'interlocuteur, de certaines caractéristiques attribuées au travailleur senior aurait pour conséquence une modification, voire un ajustement du contenu tout autant que du style de communication par ce même interlocuteur. Or, compte tenu des préjugés et croyances négatives en ce qui trait à l'avancement en âge au travail, il est plausible de penser que cet ajustement se refléterait par le biais d'un discours tout autant que des pratiques de communication âgistes. Cela dit, les études empiriques découlant des postulats de la Théorie de la communication accomodante, portant sur la mesure de la perception de communication âgiste au travail, des processus par lesquels celle-ci se concrétise et surtout de ses retombées au plan organisationnel comme individuel, sont encore peu nombreuses (Giles et Reid, 2005 ; Nelson, 2005 ; Ryan, Hummert \& Boich, 1995).

Ces lacunes ont en partie été comblées par les études quantitatives de Lagacé et al., (2008a; 2008b) menées auprès de 976 travailleurs seniors du domaine de la santé. Tel que souligné plus haut, ces études ont permis de mesurer, via questionnaire, la perception, par les seniors, de préjugés et stéréotypes âgistes. Plus encore, ces chercheurs ont évalué, sur la base des postulats de la Théorie de la communication accomodante, dans quelle mesure la communication organisationnelle, par le biais des discours et des pratiques, constitue l'un des véhicules de l'âgisme. Les résultats montrent que la communication organisationnelle s'avère bel et bien un puissant véhicule des stéréotypes et préjugés âgistes et ce, par le biais de deux axes : le discours infantilisant, voire dénigrant à l'égard du travailleur senior (par exemple, par le recours à des blagues dénigrantes sur l'avancement en âge) et les pratiques de communication visant l'exclusion de ce même travailleur (par exemple, par le refus de promotion, de formation en raison de l'âge avancé). Plus encore, ces résultats montrent aussi que la communication organisationnelle âgiste suscite le mécontentement des seniors et qu'enfin, ce mécontentement agit comme déclencheur d'une séquence de désengagement psychologique, puis effectif (l'ensemble des résultats des études est illustré à la Figure I). 


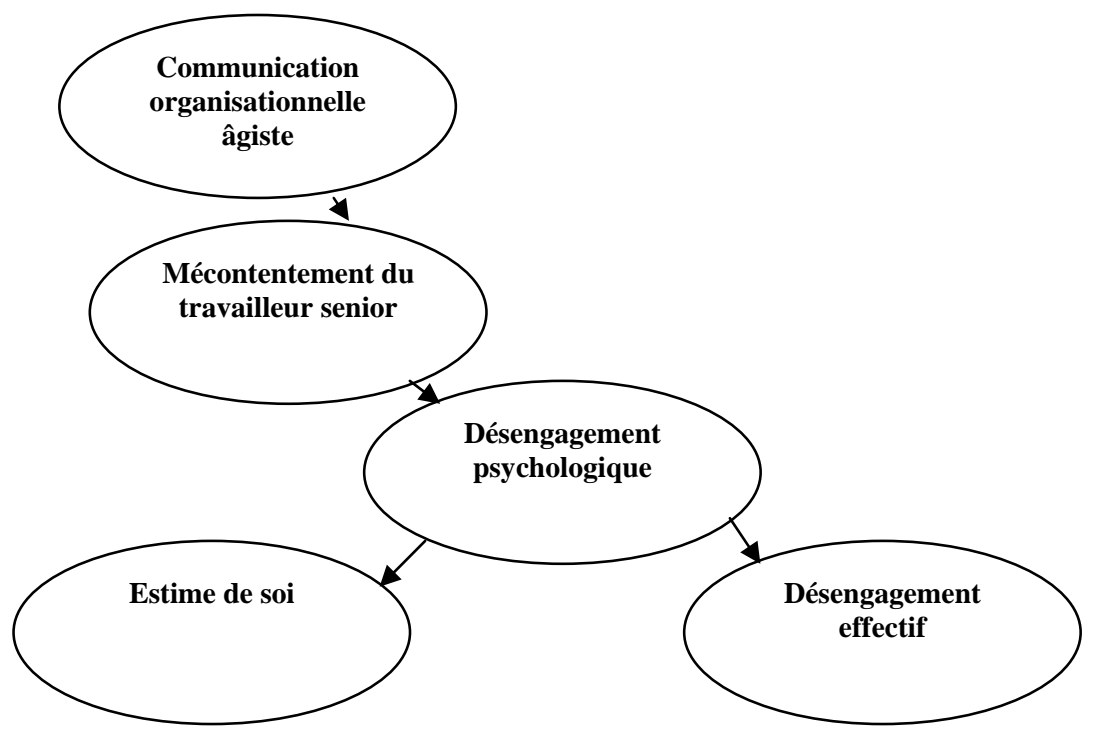

Figure 1. Modèle découlant des études de Lagacé, Tougas, Laplante, Neveu $(2008 a ; 2008 b)$

Ce faisant, ces études empiriques ont permis de circonscrire le rôle central de la communication dans les organisations de travail, en l'occurrence, dans le cas qui nous intéresse, en tant que tenant du désengagement psychologique et effectif des seniors. En corollaire, il est plausible de penser que la communication en milieu de travail, lorsqu'elle est dénuée d'âgisme, peut aussi être le point de départ de l'engagement (voire du réengagement) des seniors. Dans ce qui suit, nous explorons plus en profondeur cette dernière hypothèse.

La communication organisationnelle englobe de multiples dimensions : verbale comme non verbale, implicite comme explicite, formelle comme informelle (Laramée, 2004). Précisément, la communication en milieu de travail réfère tout autant à la nature de l'information officielle et non officielle, le dit comme le non-dit et surtout la manière, voire les stratégies selon lesquelles l'information est véhiculée et partagée. Le présent article ne permet pas de cibler toutes et chacune des dimensions de la communication organisationnelle âgiste de même que leur impact sur le désengagement des travailleurs vieillissants; cependant, en s'appuyant tout à la fois sur les postulats de la théorie de la communication accomodante (Giles \& Reid, 2005 ; Giles, Coupland 
\& Coupland, 1991) ainsi que sur résultats des études de Lagacé et al., (2008a ; 2008b), il semble tout à fait pertinent de miser sur le discours organisationnel comme l'un des points de départ, l'un des éléments moteur d'une culture de travail inclusive pour tous les âges.

Miser sur un discours organisationnel inclusif, c'est passer d'une logique de perception (par extension de gestion) du travailleur senior comme d'un "problème à gérer» à celui d'une "valeur ajoutée ». Concrètement, cela sous-entend de dépouiller les messages organisationnels incitatifs d'une sortie, d'un départ précoce de ces mêmes travailleurs, lesquels dissimulent bien souvent des préjugés âgistes : « rajeunir, regénérer la main-d'œuvre », « recruter du sang neuf », «se débarrasser du bois mort» ne sont-ils pas des exemples de discours visant l'exclusion des travailleurs seniors âgés, révélant ainsi le favoritisme de certaines générations au détriment d'une autre? Le discours, rappelons-le, est le reflet des perceptions et en cela il n'est certes pas inoffensif. Cela est d'autant plus vrai lorsque ce discours est utilisé, voire intégré, sinon même privilégié par ceux et celles au sommet d'une organisation de travail.

Les gestionnaires et la communication : remanier le discours sur le vieillissement au travail

Les gestionnaires, c'est-à-dire les supérieurs d'une entreprise donnent le ton en ce qu'ils incarnent une vision, traduisent une culture de travail, laquelle comporte une part substantielle de référents implicites. Conséquemment, le changement de mentalité nécessaire à la mise en place d'une culture de travail inclusive doit, d'abord et avant tout, être initié par ces mêmes gestionnaires et supérieurs. Faute de quoi, le discours, le message, les pratiques âgistes ne feront que s'ancrer plus solidement et ce, de haut en bas de la structure organisationnelle. Dans un contexte de pénuries de compétences tout comme de vieillissement de la main-d'œuvre, les gestionnaires d'entreprises doivent relever ce défi d'une culture de travail inclusive pour toutes les générations, lesquelles se côtoient désormais à la même table. Ces gestionnaires exercent en effet un rôle central, voire prioritaire, dans les pratiques de communication (par exemples quant au partage et à l'accessibilité de l'information, à la formation, aux promotions, etc.) tout autant que dans le discours véhiculé au sein d'une organisation. En ce sens, si les dirigeants intègrent des pratiques communicationnelles âgistes, il est plausible de penser que l'ensemble des travailleurs les endossera tout autant (à tout le moins, dans une certaine mesure). 


\section{Analyses}

Amorcer une réflexion quant au rôle que les gestionnaires d'entreprise peuvent jouer pour mettre fin à l'âgisme au travail semble d'autant plus nécessaire que certains chercheurs soutiennent que les gestionnaires seniors nourrissent eux-mêmes des préjugés et stéréotypes négatifs envers leurs employés âgés et ce, davantage qu'à l'égard de leurs plus jeunes employés (Hassel \& Perrewe, 1995). Ainsi, quoique ces employés âgés soient perçus comme productifs et fiables par leurs supérieurs seniors, ces derniers les perçoivent aussi comme des travailleurs rigides, résistant au changement et à l'innovation (Henkens, 2005), Hassel et Perrewe (1995) expliquent ce paradoxe en soutenant que les gestionnaires seniors préfèrent mettre à l'avant plan leur statut de dirigeant (plutôt que de senior), recourant à ce statut comme un «bouclier » pour se protéger des préjugés face au vieillissement en milieu de travail. Autrement dit, en se distançant de cette facette négative de leur identité sociale, ils se prémuniraient potentiellement des préjugés et stéréotypes qui y sont rattachés. Compte tenu que le vieillissement de la main-d'œuvre aura pour incidence une augmentation du nombre de gestionnaires seniors (Munnell, Sass \& Soto, 2006), les attitudes âgistes de ces mêmes dirigeants ne seront certes pas sans incidence quant à la cristallisation d'une culture des âges exclusive.

De comprendre comment les gestionnaires perçoivent eux-mêmes le vieillissement et par extension, quelle représentation ils entretiennent en regard de leurs employés seniors est un point de départ incontournable pour changer les mentalités face à l'avancement en âge au travail. Encore trop peu d'études sont menées à cet effet. De la même façon, la communication, en tant qu'élément « organisant» de l'entreprise et donc forcément en lien avec l'engagement (ou le désengagement) des travailleurs, doit être davantage circonscrite lors d'études ultérieures. Précisément, il est impératif de saisir le discours que les gestionnaires véhiculent tout autant que les pratiques de communication qu'ils favorisent quant au vieillissement au travail. En saisissant la logique de communication qui guide leur mode de gestion des seniors, il est plausible de penser que cela ouvrirait la voie à de véritables changements de culture organisationnelle. Forcément, l'instauration d'une culture de travail inclusive n'est pas strictement tributaire des pratiques de communication des gestionnaires et dirigeants d'entreprise. Cependant, à la lumière des résultats des études antérieures sur les répercussions de l'âgisme au travail (Lagacé et al., 2008a ; 2008b), force est de constater que la communication est l'un des éléments clés de la transformation des mentalités en regard de l'avancement en âge ; et c'est dans cette optique d'une exploration 
plus en profondeur de la communication âgiste au travail que nous suggérons de mieux circonscrire, lors d'études futures, le rôle des dirigeants d'entreprise quant au maintien de ce type de communication. Il s'agirait ainsi de mettre à l'épreuve le modèle illustré à la Figure II, lequel s'inscrit en extension des résultats obtenus dans le cadre des études de Lagacé et al., (2008a ; 2008b).

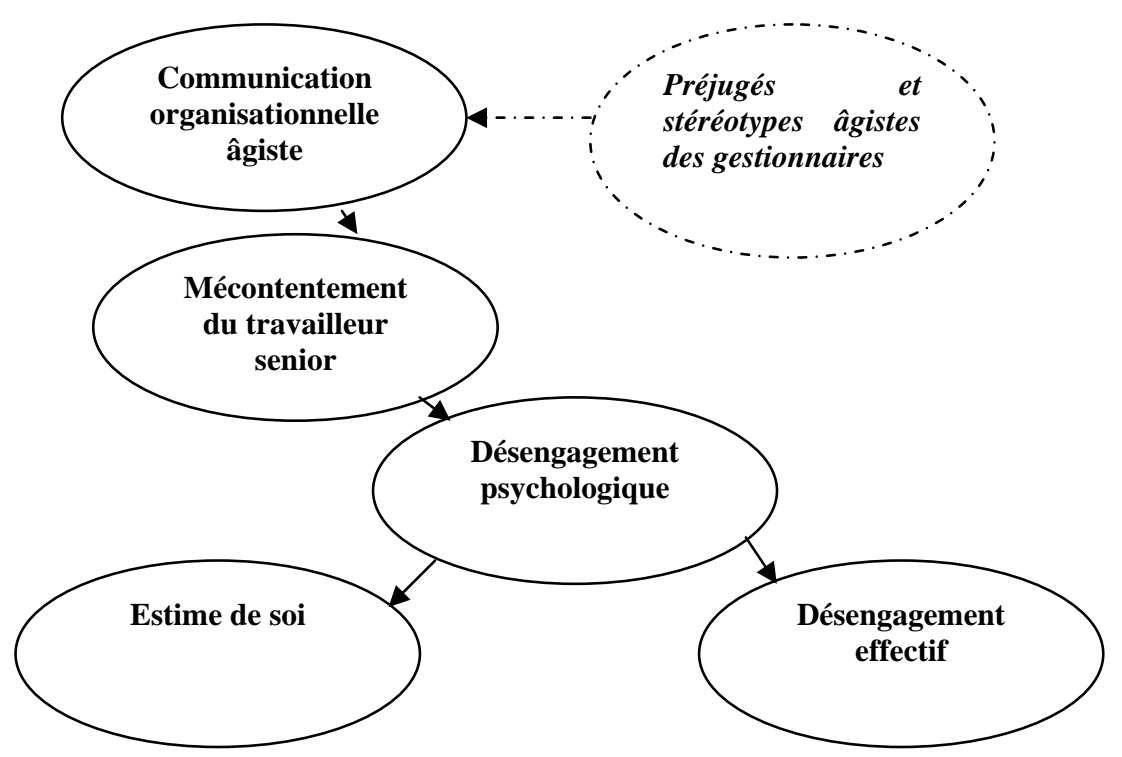

Figure 2. Modèle proposé intégrant l'évaluation du discours et des pratiques de communication des gestionnaires

Ce modèle sous-tend l'hypothèse que les dirigeants d'entreprise, lorsqu'ils endossent les préjugés et stéréotypes âgistes, risquent de s'inscrire à la source d'un processus de désengagement du travailleur senior, en ce que ces gestionnaires ont recours à une communication reflétant de tels préjugés. En corollaire et dans le cas où cette hypothèse s'avérerait confirmée, il est aussi plausible de penser que ces mêmes dirigeants seraient aussi un élément de la solution lorsqu'il s'agit de contrer l'âgisme.

Au point de vue méthodologique, compte tenu que l'ensemble des études portant sur l'âgisme ont jusqu'à maintenant privilégié une approche corrélationnelle, nous suggérons que la mise à l'épreuve de l'hypothèse ci-dessus s'effectue par le biais d'un design quasi expérimental. Ce faisant, ce type de design permettrait de tester de manière causale, dans quelle mesure la souscription, par les 
gestionnaires, à des préjugés sur la base de l'âge, contribue à la mise en place de pratiques de communication âgistes. Par exemple, la procédure constituerait à exposer, dans un premier temps, un groupe de gestionnaires à un scénario fictif au cours duquel un travailleur commet une erreur coûteuse pour son organisation (ce faisant, il pourrait s'agir d'une erreur en matière d'utilisation des nouvelles technologies de communication: ainsi, un courriel contenant de l'information secrète est envoyé par mégarde à des individus non concernés). Deux versions de ce scénario fictif seraient présentées : la première, dans laquelle l'erreur serait commise par un jeune travailleur, la seconde, par un travailleur senior. Dans un deuxième temps, les gestionnaires participants à l'étude seraient questionnés quant à leur interprétation de l'erreur et ce, en fonction de différentes possibilités : précisément, l'erreur commise pourrait être attribuée soit a) à l'âge, soit b) à des facteurs structurels en lien avec l'environnement de travail. Il est plausible de postuler que les gestionnaires souscrivant aux stéréotypes âgistes, seraient enclins à attribuer l'erreur commise par le travailleur senior à l'âge avancé de ce dernier (l'un des plus féroces préjugés âgistes au travail étant celui selon lequel les travailleurs vieillissants sont inaptes à utiliser les nouvelles technologies de communication); en revanche, pour le jeune travailleur, l'erreur serait attribuable à des facteurs structurels.

Enfin, dans un troisième temps, les gestionnaires participants seraient invités à élaborer des solutions potentielles visant à restaurer l'erreur commise et ce, en en termes de pratiques communicationnelles à l'égard du travailleur (par exemple, ces pratiques concerneraient les stratégies de partage d'information privilégiées, les types de langage utilisé, les stratégies en lien avec le plan de carrière - formation, promotion - etc.). L'hypothèse sous-jacente étant que les pratiques communicationnelles seraient tributaires du degré de souscription aux stéréotypes âgistes : plus exactement, les gestionnaires souscrivant à l'âgisme privilégieraient une communication stéréotypée sur la base de l'âge envers le travailleur senior: langage infantilisant et / ou contrôlant, accès limité à la formation, aux promotions, etc.). En somme, dans un tel cas, les pratiques communicationnelles viseraient l'exclusion implicite du travailleur senior en ce que celui-ci serait perçu comme un travailleur incompétent et désuet (et ce, sur la seule base de son âge). En revanche, le gestionnaire ne souscrivant pas aux préjugés âgistes aurait tendance à privilégier des pratiques communicationnelles dénuées de langage infantilisant et / ou contrôlant et favorisant la formation, le développement continu, l'accès à des promotions pour le travailleur et ce, quel que soit son 
âge. Bref, dans un tel scénario, l'avancement en âge ne s'inscrivant pas comme une barrière perceptuelle pour le gestionnaire, des pratiques de communication intégrantes et inclusives seraient alors privilégiées.

$\mathrm{Au}$ final, nous maintenons que ce n'est que dans un contexte de culture organisationnelle inclusive sur la base de l'âge que les structures formelles visant l'extension de la vie professionnelle (comme la planification et le développement de carrière ou encore l'aménagement du temps de travail) seront efficaces. Des chercheurs comme Guérin et Saba (2003) ont d'ailleurs identifié la communication comme l'une des pratiques sur laquelle miser pour maintenir en emploi les seniors en soulignant que : «les pratiques de communication (...) ne peuvent manquer d'influencer les perceptions qui sont à la base des sentiments d'équité et de justice et donc du désir de rester dans l'organisation. » (p. 600).

\section{Conclusion}

Bannir l'âgisme au travail n'est pas qu'une question d'équité, c'est une question de survie organisationnelle. Devant le réel défi démographique qu'est celui des pénuries de main-d'œuvre, les dirigeants doivent s'assurer d'un recrutement continu tout autant que du maintien en emploi et de l'engagement de leurs travailleurs. Et cela est particulièrement vrai pour les travailleurs seniors qui, très souvent, traduisent la mémoire, le savoir-faire et le savoir être d'une organisation; mais cette expertise acquise risque de se volatiliser si l'image négative du vieillir social, cultivée à plus grande échelle, se répercute dans les milieux de travail. En cela, le vieillissement de la main-d'œuvre peut être vu comme une catastrophe mais il peut aussi être saisi comme une occasion de réinventer le travail pour les 45-70 ans et du coup, de redonner sa pleine valeur à l'expérience. À n'en pas douter, toutes les générations de travailleurs seront gagnantes de ce remaniement de discours (ne serait-ce que du point de vue économique), par le transfert, voire le partage des connaissances qui risquent fort probablement (et naturellement) d'en découler.

\section{Bibliographie}

ASSOCIATION INTERNATIONALE FRANCOPHONE DES AÎNÉS - AIFA Colloque international : l'engagement des aînés dans la société, 2002. Disponible sur: http://www.habiterautrement.org/28 Homes/Engagement-des-aines-dans-la-societeAIFA.pdf (consulté le 13.11.2007). 
Analyses

ARROWSMITH, James, \& MCGOLDRICK, Ann E. Bridging the theory and practice divide through collaborative research - the case of ageism in employment. 1996. Working paper, British Academy of Management Conference, Lancaster, U.K.

AVOLIO, Bruce J. \& BARRETT, Gerald V. Effects of age stereotyping in a simulated interview. Psychology and Aging, 1987, vol. 2, pp. 56-63.

CONGRÈS DU TRAVAIL DU CANADA Mémoire $d u$ Congrès $d u$ travail du Canada devant le Comité permanent du développement des ressources humaines, du développement des compétences, $d u$ développement social et de la condition des personnes ayant un handicap, 2004. Disponible sur:

http ://congresdutravail.ca/updir/may4.pdf (consulté le 15.12. 2007).

CÔTÉ, Marcel. La main-d'œuvre vieillissante: une gestion à repenser. Gestion, 1991, vol. 16, pp. 9-16.

COWAN, Alison, FARQUHAR, Carolyn \& MACBRIDE-KING, Judith. Rapport $d u$ Conference Board $d u$ Canada: Building Tomorrow's Public Service Today: Challenges and Solutions in Recruitment and Retention, 2002. Disponible sur: http://www.conferenceboard.ca/documents (consulté le 19.12. 2007).

CROCKER, Jennifer, \& MAJOR, Brenda. Social stigma and selfesteem. The self-protective properties of stigma. Psychological Review, 1989. vol. 96, pp. 608-630.

CROCKER, Jennifer, MAJOR, Brenda, \& STEELE, Claude. Social stigma. In: FISKE, Susan T., GILBERT, Daniel T. \& LINDZEY, Gardner. (Coord.), Handbook of Social Psychology, vol. 2, 1998, pp. 504-553.

CUDDY, Amy J.C., FISKE, Susan T. Doddering but Dear: Process, Content, and Function in Stereotyping of Older Persons. In: NELSON, Todd D. (Coord.), Ageism. Cambridge, MA: MIT Press, 2002, pp. 326.

FINKELSTEIN, Lisa M., BURKE, Michael J., \& RAJU, Nambury S. Age discrimination in simulated employment contexts: An integrative analysis. Journal of Applied Psychology, vol. 80, 1995, pp. 652-663.

GAULLIER, Xavier. L'avenir à reculons : chômage et retraite. Paris : Éditions ouvrières, 1982, $250 \mathrm{p}$.

GAULLIER, Xavier. Le temps des retraites: les mutations de la société salariale. Paris : Seuil, 2003, 95 p. 
GILES, Howard, COUPLAND, Nikolas, \& COUPLAND, Justine. Context of accommodation: Development of applied linguistics. Cambridge, England: Cambridge University Press, 1991, 321 p.

GILES, Howard, FOX, Susan, HARWOOD, Jake, \& WILLIAMS, Angie. Talking age and aging talk: Communicating through the lifespan. In: HUMMERT, Mary Lee, WIEMANN, John M. \& NUSSBAUM, Jon F. Nussbaum (Coord.), Interpersonal communication in older adulthood: Interdisciplinary theory and research, Newbury Park, CA: Sage, 1994, pp 130-161.

GILES, Howard, \& REID, Scott A. Ageism Across the Lifespan: Towards a Self-Categorization Model of Ageing. Journal of Social Issues, vol. 61, 2005, pp. 389-404.

GLOVER, Ian A. \& BRANINE, Mohamed. The challenges of longer and healthier lives. In: GLOVER, Ian A. \& BRANINE, Mohamed. (Coord.), Ageism in work and employment. Aldershot, England: Ashgate, 2001, pp. 3-21.

GUÉRIN Gilles \& SABA, Tania. Efficacité des pratiques de maintien en emploi des cadres de 50 ans et plus. Relations industrielles, vol. 58, 2003, pp. 590-619.

HASSELL, Barbara L. \& PERREWE, Pamela L. An examination of beliefs about older workers: do stereotypes still exist? Journal of Organizational Behavior, vol. 16, 1995, pp. 457-468.

HENKENS, Kene. Stereotyping Older Workers and Retirement: The Managers' Point of View. Canadian Journal of Aging, vol. 24, 2005, pp. 353-366.

LAGACÉ, Martine, TOUGAS, Francine, LAPLANTE, J., \& NEVEU, Jean-François. La communication âgiste en milieu de travail : le point de départ vers le désengagement psychologique et effectif d'infirmières de 45 ans et plus? Soumis à La Revue canadienne de communication, 2008a.

LAGACÉ, Martine, TOUGAS, Francine, LAPLANTE, J., \& NEVEU, Jean-François. La santé en péril : les répercussions de la communication agiste sur le désengagement psychologique et l'estime de soi des infirmiers de 45 ans et plus. Sous presse: La revue canadienne du vieillissement, 2008b.

LAGACÉ, Martine, \& TOUGAS, Francine. Les répercussions de la privation relative personnelle sur l'estime de soi. Une étude du rôle du désengagement psychologique auprès de travailleurs de la santé de 
Analyses

plus de 45 ans. Cahiers internationaux de psychologie sociale, vol. 69, 2006, pp. 3-13.

LANCASTER, Lynne C., \& STILLMAN, David. When Generations collide. Who they are. Why they clash. How to solve the generational puzzle at work. New York: Harper Collins, 2002, 355 p.

LARAMÉE, Alain. La communication dans les organisations. Une introduction théorique et pragmatique. Québec: Presses de l'Université du Québec, 2004, 302 p.

MAJOR, Brenda, \& SCHMADER, Toni. Coping with stigma through psychological disengagement. In: SWIM, Janet K. \& STANGOR, Charles. (Coord.), Prejudice: The target's perspective. San Diego, US: Academic Press, 1998, pp. 219-241.

MAJOR, Brenda, \& SCHMADER, Toni. Legitimacy and the construal of social disadvantage. In: JOST, John T. \& MAJOR, Brenda. (Coord.), The psychology of legitimacy: Emerging perspectives on ideology, justice and intergroup relations. Cambridge, England : Cambridge University Press, 2001, pp. 176-204.

MALATEST, Robert A. Le vieillissement de la main-d'œuvre et les répercussions sur le développement des ressources humaines. Ottawa, Ontario: R.A. Malatest \& Associates Ltd, 2003.

McCANN, Robert M., \& GILES, Howard. Ageism in the Workplace: A Communication Perspective. In: Nelson, Todd D. (Coord.), Ageism. Cambridge, MA: MIT Press, 2002, pp. 163-199.

McDONALD, P. Lynn., \& WANNER, Richard A. Retirement in a dual economy: the Canadian case. In: Marshall, V.W. (Coord.), Aging in Canada: Social Perspectives. Toronto: Fitzhenry \& Whiteside, 1987, pp. 245-261.

MORREALE, Sherwyn P., SPITZBERG, Brian H., \& BARGE, J. Kevin. Human Communication: Motivation, Knowledge and Skills. Wadsworth, Belmont, CA, 2001, 490 p.

MUNNEL., Alicia H., SASS, Steven A., \& SOTO, Mauricio. Employer attitudes towards older workers: survey results, 2006. Disponible sur :

http ://crr.bc.edu/briefs/employer_attitudes_toward_older_workers_su rvey_results.html (consulté le 12.02.08).

NELSON, Todd D. Ageism: Prejudice against our feared future self. Journal of Social Issues, vol. 61, 2005, pp. 207-221. 
ORGANISATION DE COOPÉRATION ET DE DÉVELOPPEMENT ÉCONOMIQUE (OCDE). Vieillissement et politiques de l'emploi : Vivre et travailler plus longtemps. Paris : Les Éditions de l'OCDE, 2006, $162 \mathrm{p}$.

PALMORE, Erdman B. The Ageist Survey: First findings. The Gerontologist, vol. 41, 2001, pp. 1-3.

PALMORE, Erdman B. Ageism Survey. In: PALMORE, Erdman B., BRANCH, Laurence. \& HARRIS, Diana K. (Coord.), Encyclopedia of Ageism. Binghampton, NY: Haworth Press, 2005a, pp. 25-30.

PALMORE, Erdman B. Three decades of research on ageism. Generations, vol. 29, 2005b, pp. 87-90.

RESSOURCES HUMAINES ET DÉVELOPPEMENT SOCIAL CANADA. Document d'information sur les pénuries de main-d'œuvre actuelles et futures au Canada, 2004. Disponible sur:

http ://www.rhdsc.gc.ca/fr/publications ressources/recherche/categori es/marche travail f/sp 615 10 06/shortages.pdf (consulté le 29.01.07).

RESSOURCES HUMAINES ET DÉVELOPPEMENT SOCIAL CANADA. Document d'information sur un partenariat en vue d'étudier comme les travailleurs âgés s'adaptent à un marché du travail en évolution, 2003. Disponible sur:

http ://www.rhdsc.gc.ca/fr/sm/comm/nouvelles/2002/020715_f.shtml (consulté le 22.02.07).

RYAN, Ellen Bouchard, HUMMERT, Mary Lee, \& BOICH, Linda. Communication predicaments of aging. Patronizing ehaviour toward older adults. Journal of Language and Social Psychology, vol. 14, 1995, pp. 144-166.

SALTHOUSE, Timothy A. \& MAURER, Todd J. (1996). Aging, job performance, and career development. In: BIRREN, James E. \& SCHAIE, K.Warner. (Coord.), Handbook of psychology of aging, San Diego: Academic Press, 1996, pp. 353-364.

TAYLOR, Philip E., \& WALKER, Alain. Employers and older workers. Employment Gazette, vol. 101, 1993, pp. 371-378.

TAYLOR, Philip E., \& WALKER, Alain. The ageing workforce employers' attitudes towards the employment of older people. Work, Employment and Society, vol. 8, 1994, pp. 569-91.

TAYLOR, Philip E., \& WALKER, Alain. Intergenerational Relations in the Labor Market: The Attitudes of Employers and Older Workers. 
Analyses

In: Walker, Alain. (Coord.), The New Generational Contract: Intergenerational Relations, Old Age and Welfare. London: UCL Press, 1996, pp. 159-186.

TOUGAS, Françine., RINFRET, Natalie, BEATON, Ann. M., \& De la SABLONNIÈRE, Roxane. Policewomen acting in self-defense: Can psychological disengagement protect self-esteem from the negative outcomes of relative deprivation? Journal of Personality and Social Psychology, vol. 88, 2005, pp. 790-800.

TOUGAS, Françine, LAGACÉ, Martine, LAPLANTE, Joelle \& BELLEHUMEUR, Christian. Shielding self-esteem through the adoption of psychological disengagement mechanisms: The good and the bad news. The International Journal of Aging and Human Development, vol. 67, 2008, pp. 129-148. 